\title{
Indices for Prevalence and Incidence of Periodontal Disease
}

\author{
by Sigurd P. Ramfjord, L.D.s., M.S., PH.D., " Ann Arbor, Michigan
}

E PIDEMIOLOGICAL investigations of a disease commonly start with the study of prevalence and incidence of the disease. Prevalence refers to the number of events or defects in an individual or a population at a specified time. Incidence refers to the number of defects that may accrue or accumulate in an individual or a population during a given period of time. When the extent and distribution of the disease has been investigated, the available information is utilized in search for etiological factors and studies of the nature of the disease.

Attempts are always made to gather epidemiological data in numerical systems from which an index can be computed. An index should be quantitative since it must indicate the severity of the disease with reasonable accuracy, and it should be suitable for statistical evaluation.

OBJECTIVES FOR A SCORING SYSTEM OR AN INDEX FOR PERIODONTAL DISEASE

1. To map distribution of the disease (prevalence).

a. In population groups

b. Within each dentition

c. Around each individual tooth (mesial, distal, buccal, lingual)

2. To record the progress and behavior of the disease either by longitudinal studies of the same group or by comparing prevalence studies of various age groups within the same population (incidence).

3. To serve as a basis for evaluation of the role of various etiologic factors in the pathogenesis of periodontal disease.

4. To allow an estimate of total need for periodontal therapy in population groups.

*The University of Michigan, School of Dentistry, Ann Arbor, Michigan.
5. To acquire a basis for education of the dental profession, the public, and the governmental authorities regarding the need for attention and treatment of periodontal disease; to estimate future needs for dentists and auxiliary personnel.

6. To serve as a basis for testing and evaluation of various procedures for periodontal treatment.

7. To serve as a basis for evaluation of the effectiveness of various measures in preventing or delaying the loss of teeth from periodontal disease.

8. To serve as a basis for evaluation of measures to prevent periodontal disease.

EVALUATION OF THE LITERATURE

The literature on epidemiology of periodontal disease should be evaluated on the basis of whether or not the previously listed eight objectives have been met adequately. Furthermore, the investigations should be related to the following principles which are fundamental to any type of research.

1. The methods should be described and explained to the extent that the investigation can be duplicated by any scientist in the same field or related fields.

2. The values should be assessed in an objective and documentable way (which is very difficult in clinical research). The ability to reproduce an index or survey system depends mainly upon an exact definition of the criteria for scoring and the ability of the examiner to utilize these criteria under clinical conditions.

3. The conditions for sampling of data should be controlled; the best available scientific tools should be used by properly trained and standardized investigators.

4. Control groups or previously established and acceptable baselines have to be available. 
5. The samples should be representative and of adequate size (size depending mainly upon standard deviation and number of dependent and independent variables).

6. The experimental data should be arranged and processed in such a way that they can be readily analyzed.

When the objectives for a periodontal index and these fundamental principles for research methods are applied to the reported surveys of epidemiology of periodontal disease, it seems that none of the methods listed in the literature even approach the satisfaction of such requirements.

The most common shortcomings of epidemiologic investigations of periodontal disease may be listed as follows:

1. The criteria for scoring have been inadequately described or defined.

2. Inadequate examination methods and tools have been used.

3. Only part of the periodontium has been examined (e.g. P.M.A. index ${ }^{1}$ ).

4. The assessment of the scoring values has been inadequately documented.

5. The sampling has been inadequate or misleading (for instance, use of anterior teeth exclusively in assessment of prevalence of gingivitis in population groups ${ }^{1}$ ).

6. The presence or absence of periodontal pockets has not been related to the cementum-enamel junction or any other fixed point on the teeth.

One of the greatest problems in periodontics is the insidious, inconspicuous, and commonly asymptomatic onset and progress of destructive periodontal disease. This problem provides an understandable background for the finding that figures for prevalence of periodontal disease range from 4-5 per cent ${ }^{2}$ to $95-100$ per cent ${ }^{3}$ in similar population groups. The prevalence figures have a tendency to be much higher in surveys conducted by periodontists than reported in surveys by non-periodontists.
The best known periodontal index is Schour and Massler's P.M.A. index. ${ }^{1,4}$ Used properly by standardized examiners and applied to the entire dentition, this index seems to be acceptable for the recording of superficial gingivitis. Since it has no provision for recording of periodontal destruction, the P.M.A. index is entirely unsatisfactory for investigation of destructive periodontal disease.

During the last $5-10$ years promising attempts have been made to assess clinically and roentgenographically the distribution of insidious as well as advanced periodontal disease in some population groups. MarshallDay's clinical and radiographic surveys are very comprehensive, ${ }^{5,6}$ but his methods are somewhat cumbersome for field studies and the criteria for scoring are not always defined clearly enough to allow duplication by others. The pockets are not related to any fixed points on the teeth. This situation makes it difficult to decide whether or not there is an apparent increase in depth of the crevice because of gingival swelling or the presence of true periodontal pockets associated with the loss of periodontal support. The suggested radiographic technic is inadequate, especially for the maxillary molars (Herulf $\left.f^{7}\right)$; therefore, these teeth were excluded from the radiographic score.

McIntosh, ${ }^{8}$ Mehta, Grainger, and Williams ${ }^{9}$ have included measurements of periodontal pockets in recent investigations. The pockets were not measured or assessed from any fixed points on the teeth, so the periodontal significance of the listed pockets cannot be evaluated. With minor future adjustments their methods seem to have some merit for epidemiological surveys of periodontal disease.

A new periodontal index was suggested recently by Russell. ${ }^{10}$ In this index great significance has been given to the presence or absence of periodontal pockets, and the provision has been made to include advanced periodontal disease in the scoring system. Several papers have been published during the last two years utilizing this method. ${ }^{11,12,13}$ 
For a strictly epidemiological study of variations in distribution of periodontal disease in populations, the Russell index seems to be satisfactory; but evaluated on the basis of the previously listed periodontal objectives, the method has some serious shortcomings.

1. From publications by Russell and coworkers, ${ }^{10,11,12,13}$ it seems reasonable to assume that the total prevalence of periodontal disease is grossly underestimated (e.g. 58 per cent with normal periodontium in a group of 601 males aged between 20 and 44 years in Colorado, ${ }^{10}$ while Marshall-Day, et al, ${ }^{6}$ Mehta et al, ${ }^{9}$ and others ${ }^{14,15}$ utilizing more exacting procedures for periodontal examination have found only 3 to 15 per cent without periodontal disease in similar age groups). Inadequate examination procedures based on cursory inspection of the gingival tissues without drying off the saliva for evaluation of color, without palpation for density, and without routine probing for pockets is the understandable background for the underestimation of the prevalence.

2. The recording of periodontal pockets by this method is grossly inadequate because of unsuitable tools, lack of routine probing for pockets, and lack of orientation of pockets to the cementum-enamel junction. With superficial inspection a number of pockets will remain unrecognized unless radiographs are available. This error will be most significant in mouths exhibiting good oral hygiene and little overt inflammation.

3. Increase in mobility is not recognized until the tooth is so loose that it cannot be used for function. If mobility should be included as a factor to be recorded, it seems desirable to have some provision for recording it prior to the terminal stage of periodontal disease.

Fairly good comparability of results has been obtained in the Russell method by lowering requirements to accuracy in collecting data, but very few, if any, of the eight previously listed periodontal objec- tives can be served by this method. It can be argued that a clinical index will never be perfect and that the Russell index has certain desirable features; it is fast and easy to use, it has a fair comparability of results, and it has some provision for the recording of advanced destructive periodontal disease. To some extent the condition around every tooth in the mouth is considered, and finally, it has been used rather extensively, so some basis has been established for comparing periodontal scores from various populations.

Roentgenographic examination is a useful adjunct in surveys of periodontal conditions, but the applied technics need further improvement and standardization (Miller and Seidler, ${ }^{16}$ Herulf, ${ }^{7}$ MarshallDay et al, ${ }^{6,17}$ Belting et al, ${ }^{18}$ and others. ${ }^{19}$, ${ }^{20}$ ) These methods, without clinical examination, are totally inadequate for recording of periodontal disease in its various manifestations. Roentgenograms are excellent sources for study of the relative degree of bony destruction around various teeth in the individual dentition, and the use of roentgenograms seemingly has a tendency to increase the over-all score of periodontal disease by the detection of otherwise overlooked areas of periodontal destruction. ${ }^{10}$ In field surveys it is difficult to obtain roentgenograms, and it is felt that adequate information can be secured by exacting procedures of clinical examination, including routine probing for periodontal pockets with suitable thin instruments.

Reports on mortality of teeth (Brekhus, ${ }^{21}$ Pelton et $\mathrm{al}^{, 22}$ ) have provided some indications of the relative order of loss of teeth, and properly sampled it would yield information on the reasons for loss of teeth. This method is, of course, entirely unsatisfactory for studies of morbidity of a disease.

OWN METHOD

The World Health Organization initiated a study of the epidemiology of periodontal disease in India in 1957. As consultant for WHO, I was faced with the 
inadequacy of the available systems for recording of periodontal disease. An attempt, therefore, was made to utilize the most valuable features of the previously discussed systems and to add some new principles to compensate for the various listed inadequacies of these systems.

1. It was decided to utilize a selective sampling of teeth for the examination. The most reliable sample is always the entire population, but in most epidemiological studies today, the principles of random or selective sampling are utilized in order to save time and effort. A selective sampling has to be based on a considerable amount of available information in regard to distribution of the disease to be sampled. The pattern of distribution of periodontal disease within the dentition has been studied by numerous investigators using: a) P.M.A. index for gingival manifestations, ${ }^{23}$ b) probing and roentgenograms for the detection of formation of pockets and bone loss, ${ }^{6,7,15}, 16,17$ and c) extraction records for determination of the relative order of lost teeth. ${ }^{21}$ Several attempts also have been made to divide the mouth into segments ${ }^{14}$ or sections ${ }^{24}$ for the purpose of sampling periodontal conditions in the various areas of the mouth. Various selected segments of the mouth also have been used as representatives of the entire dentition. ${ }^{14,23,25}$

Based on these available data and clinical experience from practice and teaching, the following teeth were selected as indicators of the periodontal condition within the dentition:

Tooth number 3 (maxillary right first molar)

Tooth number 9 (maxillary left central incisor)

Tooth number 12 (maxillary left first bicuspid)

Tooth number 19 (mandibular left first molar)

Tooth number 25 (mandibular right central incisor)
Tooth number 28 (mandibular right first bicuspid)

It is felt that a thorough examination of the periodontal status of these six teeth will provide a valid basis for an evaluation of the periodontal condition of the individual.

2. The second important new principle is to record the depth of the crevice or pocket in relation to the cementum-enamel junction. Measuring of the depth of the crevice or pocket has limited value unless the measurements are related to fixed landmarks on the teeth. In order to establish whether or not the periodontal disease has progressed when an eventual re-examination is completed, it is imperative that the available measurements are related to fixed landmarks on the teeth. Particularly when roentgenograms are not available, it is important that the bottom of the pocket is recorded accurately in relation to the cementum-enamel junction. Procedures for examination of pockets, position, and type of periodontal probes are technical problems of utmost importance to the reliability of the recorded data. The available periodontal probes were found to be too thick for probing of narrow pockets, so a new probe had to be constructed (" $U$. of Mich. \# 0 "). It was attempted to make this probe as thin as possible and to give it the most versatile angulation for universal probing of periodontal pockets. The location of the cementum-enamel junction necessitates removal of calculus if present at this area. It also requires some appropriate instruction and experience to be able to locate this landmark consistently. When the epithelial attachment is entirely on enamel, the cementum-enamel junction cannot always be felt by the probe; but the significant observation then is the absence of permanent periodontal destruction in spite of deep gingival crevices at times.

3. The recording of gingivitis is based on a combination of the P.M.A. index and the Russell index, but the procedures of examination are more detailed than in either 
of these methods. The field should be dried to evaluate gingival color. Palpation, probing, and observation should be combined in order to evaluate form, density, and tendency of the gingival tissues to bleed. The presence or absence of gingivitis should be determined by the combined findings of color, form, density, and tendency by the gingival tissues to bleed from palpation and probing. It is extremely easy to overlook early gingivitis; therefore the adoption of these rigid procedures of examination.

4. Calculus and plaques are scored in the search for etiologic factors. Detailed information on the presence of calculus and plaques also is of great importance in the determination of therapeutic needs as well as in evaluation of prophylactic and prevention measures.

5. Attrition is scored as a possible indicator of functional or dysfunctional activity.

6. Mobility is an indication of the functional stress applied on the tooth in relation to its support. To a certain extent mobility is indicative of the degree of periodontal destruction.

7. Lack of contact may be a factor in the etiology of periodontal disease. It may also indicate malposition or drifting of teeth.

The possibility of including malocclusion in the scoring system was considered, but it was not possible to establish acceptable criteria for numerical scoring of malocclusion.

The clinical findings are recorded on the sample sheet (Figure 1). The record also can be printed on a card with the three maxillary teeth on one side and the three mandibular teeth on the other side of the card. The procedures and criteria for the clinical recording follow:

INSTRUCTIONS FOR CLINICAL EXAMINATION

The areas surrounding teeth number 3 , 9, 12, 19, 25, 28 should be examined. Dry the field to be examined with cotton. Ob- serve and test the gingival areas around each of the above-mentioned teeth for deviations from health in color, form, density, and bleeding tendency.

Record gingival findings:

G 0 : Absence of inflammation

G 1: Mild to moderate inflammatory gingival changes not extending all around the tooth

G 2 : Mild to moderately severe gingivitis extending all around the tooth

G 3 : Severe gingivitis characterized by marked redness, tendency to bleed, and ulceration

Record calculus:

C 0 : Absence of calculus

C 1: Supragingival calculus extending only slightly below the free gingival margin (not more than $1 \mathrm{~mm}$.)

C 2 : Moderate amount of supra and subgingival calculus, or subgingival calculus only

C 3 : An abundance of supra and subgingival calculus

Record pockets:

The distance from the free gingival margin to the cementum-enamel junction and the distance from the free gingival margin to the bottom of the gingival crevice or pocket should be recorded for the mesial, the buccal, the distal, and the lingual aspects of each tooth examined. The interproximal recording should be secured at the buccal aspect of the interproximal contact areas with the probe pointing in the direction of the long axis of the tooth.

A. If the gingival margin is on enamel:

1. Measure from gum margin to cementumenamel junction and record the measurement on the crown of the schematic tooth. If the epithelial attachment is on the crown and the cementum-enamel junction cannot be felt by the probe, record the depth of the gingival crevice on the crown.

2. Measure from the gingival margin to the bottom of the pocket when the crevice extends apically to the cementum-enamel junction, the measurement should be recorded on the root of the schematic tooth. (The distance from the cementum-enamel junction to the bottom of the pocket can then be found by subtracting measurement number 1 from measurement number 2.)

B. If the gingival margin is on cementum:

1. Measure from the cementum-enamel junction to the gingival margin. Record as minus value on the root of the schematic tooth.

2. Measure from the cementum-enamel junction to the bottom of the gingival crevice. Record value on the root. 


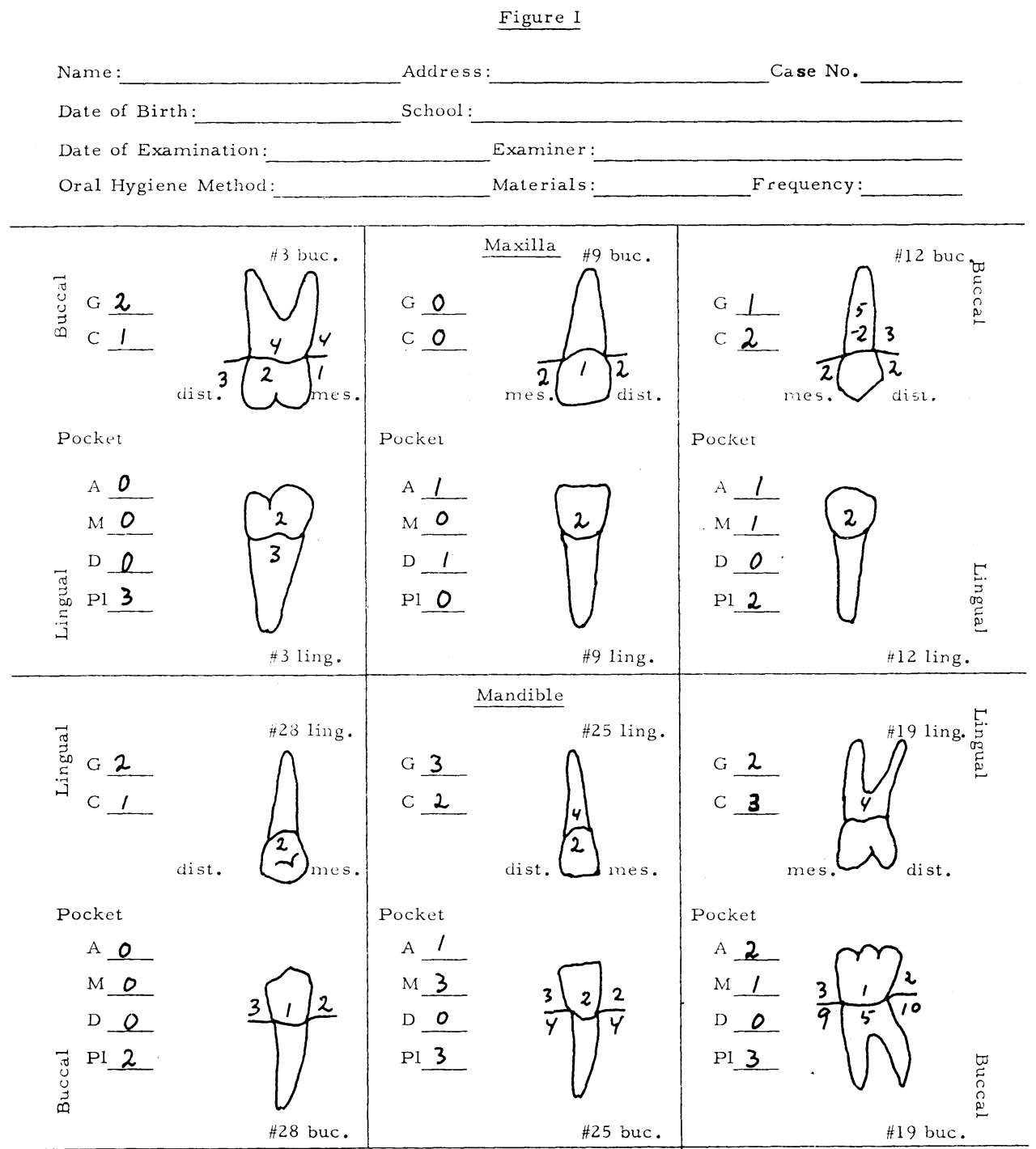

Comments:

Record occlusal and incisal attrition:

A 0 : No attrition

A 1: Attrition of enamel only; no exposure of dentin

A 2 : Attrition extends through the enamel into dentin in cuspal and incisal areas, but cuspal pattern has been maintained

A 3 : Extreme attrition; the occlusal surfaces are worn flat and "inverted" cusp pattern is present
Record mobility:

M 0 : Physiologic mobility; firm tooth

M 1 : Slightly increased mobility

M 2 : Definite to considerable increase in mobility, but no impairment of function

M 3 : Extreme mobility; a "loose" tooth that cannot be used for normal function

Record lack of contact:

D 0 : Normal contact; not open 
D 1: Opening less than $1 \mathrm{~mm}$.

D 2 : Opening between 1 and $3 \mathrm{~mm}$.

D 3 : Opening more than $3 \mathrm{~mm}$.

The side of the tooth that has the greatest interproximal opening is measured. If the adjacent tooth is missing, that area is not measured.

Record plaque after application of disclosing solution:

P 0 : No plaque present

P 1 : Plaque present on some but not on all of the interproximal and gingival surfaces of the tooth

P 2: Plaque present on all interproximal and gingival surfaces, but covering less than one half of entire clinical crown

P 3 : Plaque extending over all interproximal and gingival surfaces covering more than one half of the entire clinical crown

Only fully erupted teeth should be recorded and missing teeth should not be substituted for their examination. Any effective disclosing solution can be used for location of the bacterial plaques on the teeth. The extent of plaques cannot be evaluated without the use of the disclosing solution, which should be used as the final step of the procedures of examination. It should be applied to all of the teeth to be examined at the same time.

The recorded data can be utilized as a basis for individual index of periodontal disease. Indices for calculus, plaques, mobility, and attrition can be computed. The individual index for periodontal disease is obtained in the following manner:

First, a periodontal score for disease is tabulated for each of the examined teeth. If the gingival crevice in none of the measured areas extends apically to the cementum-enamel junction, the recorded score for gingivitis is the score for periodontal disease for this tooth. If the gingival crevice in any of the four measured areas extends apically to the cementum-enamel junction but not more than 3 millimeters (including $3 \mathrm{~mm}$.) in any area, the tooth is assigned a score for periodontal disease of 4 ; the score for gingivitis for that tooth then is disregarded in the final index for periodontal disease. If the gingival crevice in any of the four recorded areas of the tooth extends apically from 3 to 6 millimeters (including $6 \mathrm{~mm}$.) in relation to the cementumenamel junction, then the tooth is assigned a score for periodontal disease of 5 (the gingivitis score also is disregarded here). Whenever the gingival crevice extends more than 6 millimeters apically to the cementum-enamel junction in any of the measured areas of the tooth, the score of 6 is given as score of periodontal disease for that tooth (again disregarding the gingivitis score).

In this system, as in the Russell index, ${ }^{10}$ the numerical values for the scores of periodontal disease are increased in relation to the increasing loss of periodontal support. Whether or not the periodontal support is lost because of periodontitis or atrophy is not considered in this index for periodontal disease. Following the tabulation of the scores for periodontal disease for each of the examined teeth, these scores are added and the sum divided by the number of teeth examined. This division will provide the index for periodontal disease in that individual. Essentially, the method of indexing follows Russell's principles. If some of the teeth which were scheduled to be examined were missing or unerupted so that they could not be examined, the individual scores for each of the examined teeth should be added and divided by the number of teeth examined (if only four teeth were examined, the individual score of periodontal disease is added for each of these four teeth and divided by four to arrive at the index of periodontal disease for that individual.

Indices for calculus, attrition, mobility, contact, and plaques are tabulated in the same manner. For instance, the scores on calculus for each individual tooth examined are added and the sum divided by the number of teeth examined to yield the index on calculus. The scores for plaque for each tooth examined are added and divided by the number of examined teeth, and the index of plaque or oral hygiene is achieved. In the same manner indices for attrition, mobility, and contact can be achieved.

The data then are ready for analysis and statistical evaluation. Interrelation between any of the scored factors can be tested and evaluated statistically. 
Distribution and progress of periodontal disease around the individual tooth mesially, buccally, distally, and lingually also can be studied from these records.

The data recorded on the chart shown in Figure 1 can be used to illustrate the procedures of computation. The score for periodontal disease of each tooth from Figure 1 would be as follows:

For tooth number 3,4

For tooth number 9,0

For tooth number 12, 5

For tooth number 19, 6

For tooth number 25, 4

For tooth number 28, 2

The sum of these individual scores is 21 , and divided by 6 (the number of teeth examined) would yield an index of periodontal disease of 3.5 for this individual. The sum of the scores on calculus would be 9, and divided by 6 would yield an index on calculus of 1.5. The index on attrition is 0.8 ; the index for contact is 0.2 ; the index for plaque is 2.2 .

Using but one decimal point in tabulation of individual indices and raising or lowering to the nearest fraction of hundreds, one reduces the decimal; for instance, one divided by six is recorded as 0.2 and two divided by six is recorded as 0.3 . For computation of scores on population two decimal places should be used.

\section{SUMMARY}

A quantitative method of scoring periodontal disease and related conditions has been described. For each individual it requires a thorough examination of six sample teeth and surrounding tissues. Periodontal pockets are related to the cementumenamel junction to provide the periodontal significance of the measurements. Various etiologic factors are recorded. All recordings are completed in numerical systems to facilitate indexing and analysis; ambiguous criteria have been avoided; the method is easy to learn, and the comparability of examiners has been as good as can be expected from any clinical periodontal in$\mathrm{dex} ;{ }^{26}$ minimum equipment is needed; and the method is quickly applied-less than five minutes for each mouth.

An attempt has been made to meet the various requirements for a system of periodontal scoring applicable to epidemiological, clinical, and combined epidemiological and clinical investigations.

A detailed analysis of the clinical tests of this method will be published in a few months through the World Health Organization.

\section{ACKNOWLEDGMENTS}

Valuable advice has been received from Drs. A. L. Russell and C. D. Marshall-Day. All members of the World Health Organization Periodontia Seminar in Bombay in 1957 were extremely helpful in working out details of this method. Dr. C. L. Sebelius, of the World Health Organization, has been a consistent source of advice and guidance throughout this investigation.

\section{BIBLIOGRAPHY}

1. Schour, T., and Massler, M. Gingival Disease in Postwar Italy. 1. Prevalence of Gingivitis in Various Age Groups. J.A.D.A. 35:475-482, Oct. 1947.

2. Messner, C. T., et al. Dental Survey of School Children Ages 6-14 Years. Made in 26 States. Pub. Health Bul. No. 226, 1936.

3. McCall, J. O. The Periodontist Looks at Children's Dentistry. J.A.D.A. 20:1518-1521, Aug. 1933.

4. Massler, M., et al. Gingivitis in Young Adult Males: Lack of Effectiveness of a Permissive Program of Toothbrushing. J. Periodont. 28:111-124, Apr. 1957.

5. Marshall-Day, C. D. The Epidemiology of Periodontal Disease. J. Periodont. 22:13-23, Jan. 1951.

6. Marshall-Day, C. D., Stevens, R. G., and Quigley, L. F., Jr. Periodontal Disease Prevalence and Incidence. J. Periodont. 26:185-203, July 1955.

7. Herulf, G. On the Marginal Alveolar Ridge in Students. Acta Genetic. et Statistic Med. 2:256288, 1951 .

8. McIntosh, W. G. Gingival and Periodontal 
Disease in Children. Canad. Dent. A. J. 20:12-16, Jan. 1954.

9. Mehta, M. M., Grainger, R. M., and Williams, C. H. M. Periodontal Disease Among Adults. Canad. Dent. A. J. 21:617-624, Nov. 1955.

10. Russell, A. L. A System of Classification and Scoring for Prevalence Surveys of Periodontal Disease. J. Dent. Res. 35:350-359, June 1956.

11. Russell, A. L. Some Epidemiological Characteristics of Periodontal Disease in a Series of Urban Populations. J. Periodont. 28:286-293, Oct. 1957.

12. Benjamin, E. M., Russell, A. L., and Smiley, R. D. Periodontal Disease in Rural Children of 25 Indiana Counties. J. Periodont. 28:294-298, Oct. 1957.

13. Russell, A. L. A Social Factor Associated with the Severity of Periodontal Disease. J. Dent. Res. 36:922-926, Dec. 1957.

14. King, J. D. Gingival Disease in Dundee. D. Record. 65:9-16, 32-38, 55-60, 1945.

15. Lovdal, A., Arno, A., and Waerhaug, J. Incidence of Clinical Manifestations of Periodontal Disease in Light of Oral Hygiene and Calculus Formation. J.A.D.A. 56:21-33, Jan. 1958.

16. Miller, S. C., and Seidler, B. B. Relative Alveoloclastic Experience of the Various Teeth. J. Dent. Res. 21:365-371, Aug. 1942.

17. Marshall-Day, C. D., and Shourie, K. L. Roentgenographic Survey of Periodontal Disease in India. J.A.D.A. 39:572-588, Nov. 1949.
18. Belting, C. M., Massler, M., and Schour, T. Pervalence and Incidence of Alveolar Bone Disease in Men. J.A.D.A. 47:190-197, Aug. 1953.

19. Sandler, H. C., and Stahl, S. S. The Influence of Generalized Disease on Clinical Manifestations of Periodontal Disease. J.A.D.A. 49:656-667, Dec. 1954.

20. Blacharsh, C., Staub, D., and Margolis, R. Some Alveolar Bone Findings in Hospitalized $\mathrm{Pa}-$ tients. J. Periodont. 29:53-58, Jan. 1958.

21. Brekhus, P. J. Report on a Statistical Investigation on the Loss of Human Teeth. J.A.D.A. 15:679-690, Apr. 1928.

22. Pelton, W. J., Pennell, E. H., and Druzina, A. Tooth Morbidity Experience of Adults. J.A.D.A. 49:439, Oct. 1954 .

23. Massler, M., Schour, T., and Chopra, B. Occurrence of Gingivitis in Suburban Chicago School Children. J. Periodont. 21:146-164, July 1950.

24. Mehta, I. S., et al. Prevalence of Periodontal (Parodontal) Disease 5. Epidemiology in Indian Child Population in Relation to Their Socio-Economic Status. Internat. D. J. 6:31-40, Mar. 1956.

25. Parfitt, G. J. A Five Year Longitudinal Study of the Gingival Condition of a Group of Children in England. J. Periodont. 28:26-32, Jan. 1957.

26. Sandler, H. C. Testing the Uniformity of the P.M.A. Index as a Measurement of Periodontal Disease. J. Dent. Res. 31:323-325, June 1952.

\section{ALPHA OMEGA FRATERNITY}

"Total Periodontal Concept" is the theme of the premiere Sunshine Seminar to be presented by the Alumni of the Greater Miami Chapter of Alpha Omega Fraternity. This initial program will be at the Deauville Hotel, Miami Beach, from January 25 through January 30, 1959. Clinicians include Drs. Morris Amsterdam, Walter Cohen, Don McQueen, Balint Orban and John Pritchard.

Complete information about this program may be obtained by writing Dr. Irving Gordon, 420 Lincoln Road, Miami Beach 39, Florida. 